\title{
ORIENTATION OF FIRST-RANKED GALAXIES IN RICH CLUSTERS
}

\author{
GEORGE RHEE and NICO ROOS* \\ Sterrewacht Leiden, The Netherlands
}

\begin{abstract}
We present new evidence that first ranked galaxies are aligned with their parent cluster and with the direction of the nearest neighbour cluster (scale $15 h^{-1} \mathrm{Mpc}$ ). The effect is stronger for $\mathrm{cD}$ and $\mathrm{gE}$ galaxies than for first-ranked galaxies of later type. The relevance of this result for different galaxy formation scenarios is discussed. In hierarchical clustering scenarios like the cold dark matter theory, galactic halos and clusters of galaxies are expected to have moderate asphericity. We present some numerical results of an on-going study of the dissipationless collapse of moderately aspherical systems. Our results indicate that the central part of the collapsed and virialized system does show the large scale elongation imposed by the initial conditions. It is pointed out that this may have important implications also for the properties of disk galaxies in dark halos.
\end{abstract}

\section{Introduction}

The orientation of galaxies with respect to each other and with respect to the large-scale structure of which they are members may contain important information about the formation of galaxies and clusters of galaxies. Galaxies and clusters are generally thought to form as a result of gravitational amplification of small fluctuations in the primordial distribution of matter (see, however, Ostriker and Cowie, 1981). Density fluctuations on scales smaller than clusters are expected to have higher initial amplitudes but are more vulnerable to damping processes in the early universe. As a result there are two types of galaxy formation scenarios differing in the order in which galaxies and clusters form. In pancake (adiabatic, hot dark matter) scenarios, fluctuations on the scale of galaxies are damped and clusters form before galaxies. Galaxies are then likely to carry the imprint of the anisotropy in the large-scale structure of which they are formed and orientation effects are expected (e.g., Djorgovski, 1987; Zel'dovich et al., 1982). On the other hand in hierarchical clustering (isothermal, cold dark matter) scenarios galaxies form first and clusters thereafter via continuous gravitational clustering on larger scales. In this scenario most galaxies or clusters, but orientation effects may not be completely absent. Firstly, the angular momentum of disk galaxies results from tidal torques exerted by nearby protogalaxies prior to disk formation. This may yield orientation correlations on small scales. Secondly, not all galaxies are formed before clusters. Some fraction will, for example, grow by mergers during and after the collapse of clusters. Information about the large-scale galaxy distribution may somehow be remembered by these galaxies.

* Astronomical Institute of the Catholic University of Nijmegen, The Netherlands.

Astrophysics and Space Science 157: 201-210, 1989.

(C) 1989 Kluwer Academic Publishers. Printed in Belgium.

(C) Kluwer Academic Publishers • Provided by the NASA Astrophysics Data System 
During the last decades several alignment effects between galaxies and clusters have been reported and subsequently disputed (see Djorgovski, 1987, for a review). One alignment effect has survived and seems now well established: the alignment of firstranked galaxies with their parent cluster and even with the surrounding galaxy distribution on scales up to $15 h^{-1} \mathrm{Mpc}$ (Sastry, 1968; Carter and Metcalfe, 1980; Binggeli, 1982; Struble and Peebles, 1985; Rhee and Katgert, 1987; Lambas et al., 1988). We find an effect which appears to extend over 3 decades in distance from the first-ranked galaxy and provides a new and important clue to the formation of galaxies and clusters.

In the present paper we first present the latest results of our study of a complete sample of 108 Abell clusters with richness $R>0$ and redshift $z<0.1$ (Section 2). In Section 3 we discuss the formation of $\mathrm{cD}$ galaxies, and in Section 4 we present a first result of $N$-body simulations bearing on the dissipationless formation of moderately aspherical halos and clusters of galaxies in hierarchical clustering scenarios. Our results are discussed and summarized in Section 5.

\section{Observations and Results}

\subsection{OBSERVATIONS}

The automatic plate-scanning machine of Leiden Observatory (Swaans, 1981) is used to study a complete sample of 108 Abell clusters $(R>0, z<0.1)$. All objects with $m_{R} \sim 19$ within a circle of diameter $2 h^{-1} \mathrm{Mpc}$ centred on the cluster centre (cD galaxy or gravity centre of the central concentration of galaxies) are detected and discriminated from stars using Kron's discriminator $r_{-1}$. The results are then checked by visual inspection. Typically 100-300 galaxies are detected per cluster. From the azimuthal distribution of galaxies in the circular aperture we determined the amplitude and phase of a $180^{\circ}$-component, using Fourier sums (see Rhee and Katgert, 1987). These two parameters were calculated for several sets of $N$ brightest galaxies (with $N=i \times 10$ ), in order of decreasing brightness, and for 'even' and 'odd' subsets thereof (with $j=2,4, \ldots$. and $k=1,3, \ldots)$ (see Figure 1). If the phase is, to within the statistical uncertainties, equal for even and odd subsets and more or less constant with increasing $N$, and if the amplitude is sufficiently high, we consider the cluster elongated, with the position angle of symmetry equal to the phase of the $180^{\circ}$-component. With these criteria $55 \%$ of our clusters are clearly and consistently elongated in the central $2 h^{-1} \mathrm{Mpc}$ diameter area, with uncertainties in the position angle less than $15^{\circ}$.

\subsection{Results}

Two types of alignment effects will be presented in this section:

(i) the alignment of first ranked galaxies with their parent cluster and with the direction towards the nearest neighbour cluster, and

(ii) the alignment of the cluster X-ray image with the first-ranked galaxy and with the galaxy distribution.

In Figure 2(a) we show the histogram of the differences between the two position 


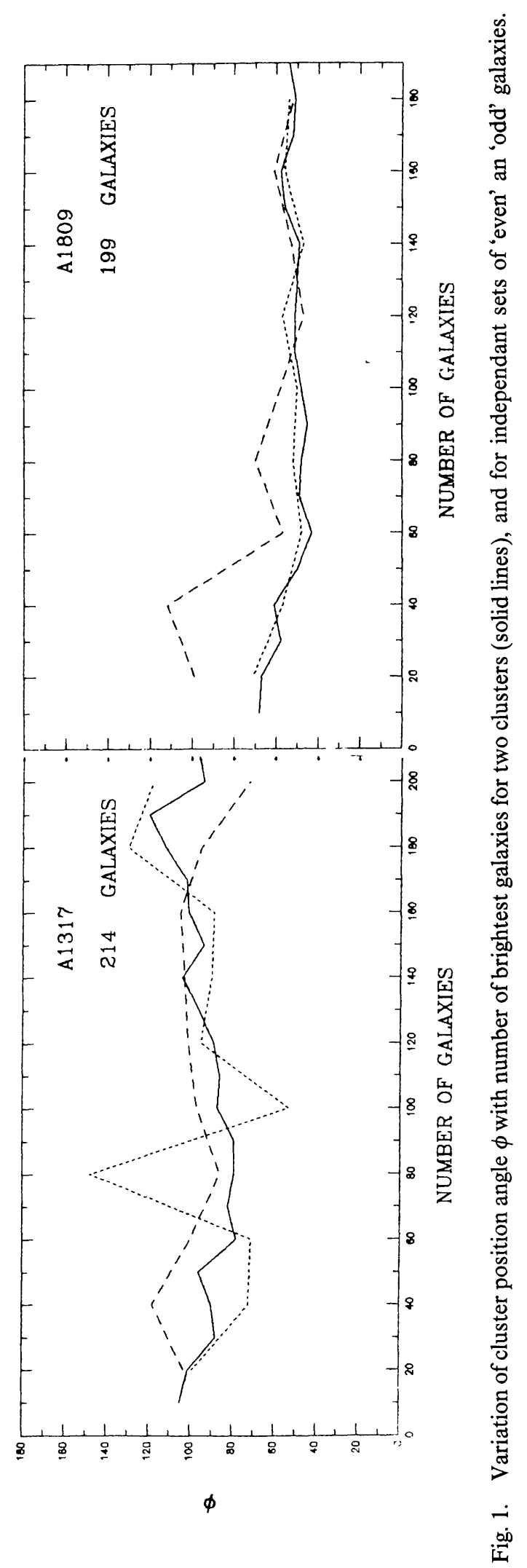




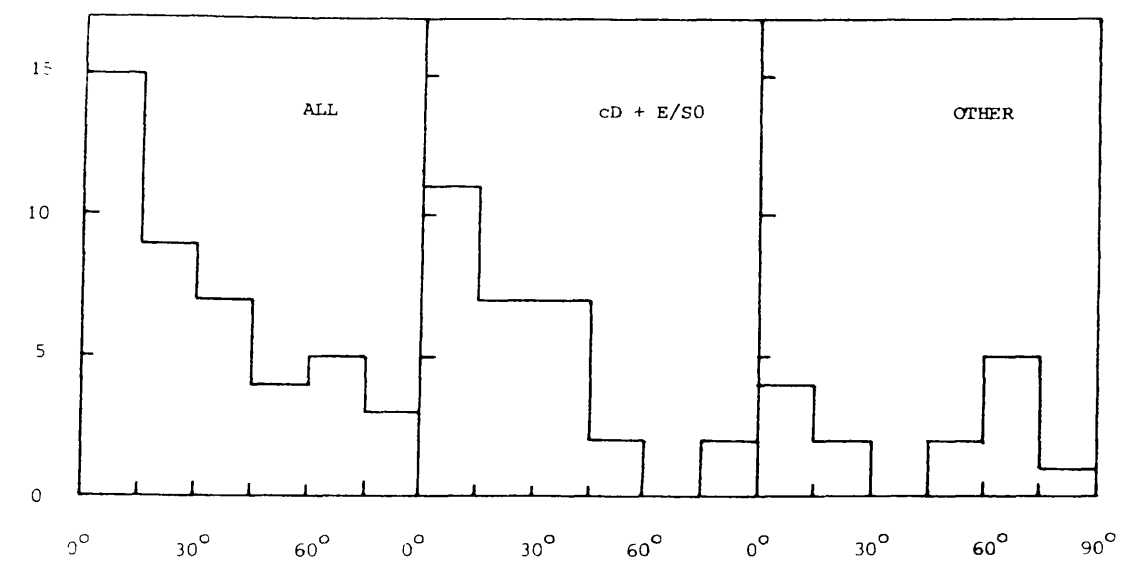

Fig. 2. Histograms of the difference between the position angle of the first-ranked galaxy and the elongation direction of the galaxy distribution for 43 significantly elongated clusters.

angles for all 43 clusters that are clearly elongated and for which the position angle of the first ranked galaxy has been published. Figures 2(b) and 2(c) show the same but for different classes of first-ranked galaxy. Clearly there is an effect which is almost entirely attributable to $\mathrm{cD}$ and $\mathrm{E} / \mathrm{S} 0$ first-ranked galaxies.

Rich clusters are strongly clustered on scales $40 h^{-1} \mathrm{Mpc}$ and alignment effects may extend to much larger scales than a few Mpcs. Binggeli's original result on the orientation of clusters with respect to their neighbours could not be confirmed (Struble and Peebles, 1985; Rhee and Katgert, 1987). On the other hand there is now firm evidence that first-ranked galaxies do know the orientation of the surrounding galaxy distribution up to a scale of $15 h^{-1} \mathrm{Mpc}$ (see Figures 3(a) and 3(b), cf. Lambas et al., 1987).

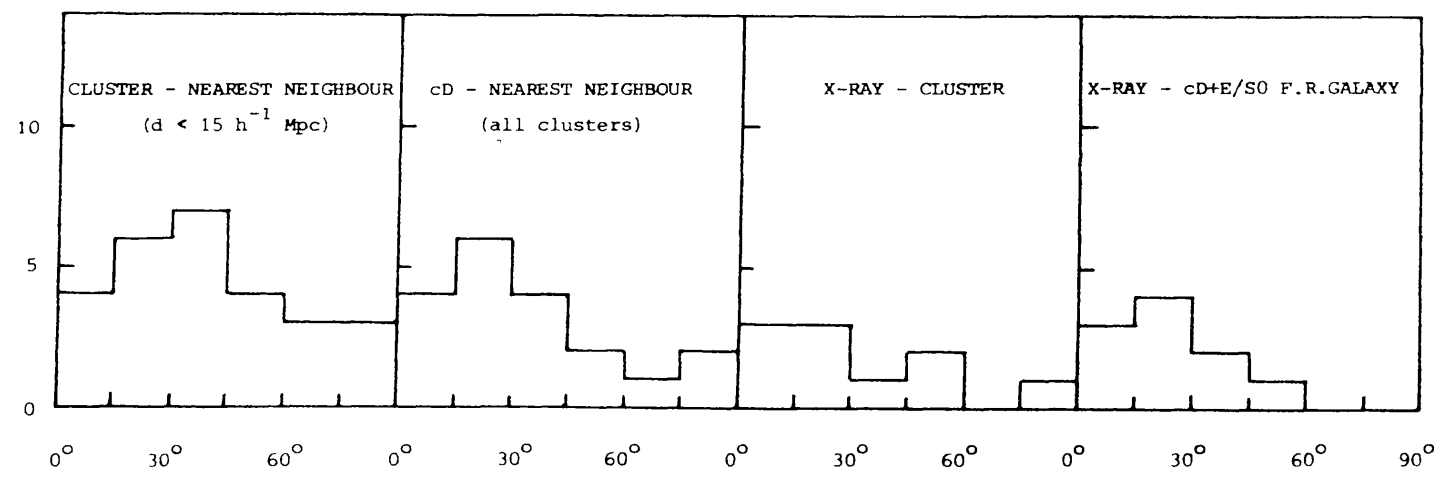

Fig. 3. Histograms of the differences between first-ranked galaxy position angle and galaxy distribution elongation angle on the one hand, and direction to nearest neighbour and elongation direction of $\mathrm{X}$-ray surface brightness on the other.

Of the clusters in our sample with reliable position angles for first ranked (cD!) galaxies and galaxy distributions, ten have Einstein IPC-observations with sufficient signal from which we could determine the elongation of the X-ray surface-brightness. The first-ranked galaxy appears to align very well with the hot gas (Figure 3(d)), whereas there may also be an alignment between galaxy distributions and hot gas but less strongly 
than for the first-ranked galaxies. Beers and Tonry (1986) in their study of the central density of rich clusters have shown that for a sample of 48 clusters the median offset between the X-ray and $\mathrm{D} / \mathrm{cD}$ centres is $70 h^{-1} \mathrm{kpc}$. The median offset of the median cluster centres with respect to the X-ray and $\mathrm{D} / \mathrm{cD}$ centres is $120 h^{-1} \mathrm{kpc}$ and $90 h^{-1} \mathrm{kpc}$, respectively. The fact that the first-ranked galaxy is well aligned with the hot gas and that the centres correspond so closely suggests a physical connection between the X-ray emission in the cluster centre and the first-ranked galaxy. The only weak alignment between the X-ray emission and the galaxy distribution may be due to the fact that the potential is less elongated than the resulting mass distribution and that cluster position angles cannot be determined as accurately as first-ranked position angles.

\section{Formation of First-Ranked Galaxies in Rich Clusters}

Knowledge of the orientation of the large-scale galaxy distribution seems to be limited to the first-ranked galaxy in rich clusters. This indicates that these galaxies were formed during or after the formation of their parent cluster, for instance via galaxy mergers. In hierarchical clustering scenarios the collapse and virialization of clusters proceeds via the merging of already collapsed substructures such as galactic halos and subclusters of galaxies (White, 1976; Cavaliere et al., 1986). Most of the smaller dissipationally formed luminous galaxies may become detached from their halos (White and Rees, 1978), but the generally more massive galaxies in the cores of subclusters tend to join the merging process. As larger units virialize these galaxies may also acquire appreciable random velocities in the cluster and become detached from their halo as well. Finally only the central, massive cannibal will have a mass distribution continuous with that of the cluster. Merging of galaxies thus preferentially occurs among the more massive galaxies in regions of high galaxy density (see also Carlberg and Couchman, 1988, preprint). If one identifies merger remnants with elliptical galaxies (Toomre and Toomre, 1972) and the spheroidal components of disk galaxies (Roos, 1981) one can naturally account for the density-morphological type relation found by Dressler (1980). Densitydependent merger rates may also provide the bias in clustering of bright galaxies which is required in $\Omega=1$ cold dark matter scenarios (Carlberg and Couchman, 1988).

In this picture it is perhaps not surprising that orientational memory is preserved by first-ranked galaxies only. However, the question remains by which physical mechanism the large-scale orientation is communicated to the central part of the cluster on a scale 3 orders of magnitude smaller (note that we are dealing with the first-ranked galaxy here, not with its extended envelope). Somehow this must be due to the interplay (via mergers) between galactic orbits in an aspherical cluster and stellar orbits in the central cannibal. As a first step towards understanding this effect we will investigate the evolution of small and large-scale anisotropy in an initially homogeneous, moderately aspherical, dissipationless system. 


\section{Dissipationless Collapse of Moderately Aspherical Systems}

\subsection{INTRODUCTION}

Overdense regions in the early universe will generally not be spherically symmetric. Small deviations from sphericity tend to grow (Lynden-Bell, 1964; Icke, 1984). In cosmological CDM simulations the collapsed and virialized systems generally are triaxial having typical axial ratios of about 2 (Frenk et al., 1988; Barnes and Efstathiou, 1987). The asphericity is probably due to the initial conditions, although the radial orbit instability might also play a role (e.g., Barnes, 1985; Merritt, 1987). The basic question we would like to answer is: under what initial conditions will the initial large-scale anisotropy be communicated to the innermost regions of the collapsed and virialized system. In this section we present the first result of an on-going study aimed at this problem. Several authors have performed collapse and relaxation experiments starting from spherical (van Albada, 1982; McGlynn, 1984; May and van Albada, 1984) or from strongly aspherical triaxial configurations (Aarseth and Binney, 1978; Wilkinson and James, 1982; McGlynn, 1984; Villumsen, 1984). The second type of simulations is thought to apply to the formation of galaxies from flat sheets (or filaments) of stars or galaxies resulting from dissipational collapse. The main result of these simulations is that the final system remembers the shape of the initial configuration, although the relation between the initial and final flattening may not be unique (Aarseth and Binney, 1978). The simulations also indicate that the final ellipticity of the relaxed systems varies only little with radius, over almost an order of magnitude in radius (cf. Villumsen, 1984).

\subsection{NUMERICAL SIMULATIONS}

In our first numerical experiment we placed 4096 particles randomly inside an ellipsoid having axial ratios $2: 1: 1$. The system starts from maximum expansion and particles have small initial velocities taken at random from a isotropic Gaussian velocity distribution with a dispersion such that the initial ratio of kinetic over potential energy is 0.05 (no rotation). The equations of motion were integrated on the Leiden Convex computer using the hierarchical tree code developed by Barnes and Hut (1986). The $N$-body system was evolved for 1200 time-steps covering about $4 t_{\text {coll }}=G M^{5 / 2} /(-2 E / 3)^{3 / 2}$, which is 16 in our dimensionless units of $G=M=-(20 / 3) E=1$, where $G$ is the gravitational constant; $M$, the total mass; and $E$, the total energy of the system. Total energy was conserved to within $1 \%$. Note that in cosmological simulations the asphericity of overdense regions at maximum expansion (which will generally occur at different times along the three principal axes) may be comparable to that of our initial configuration. In Figures $4(\mathrm{a}-\mathrm{d})$ the evolution of the $N$-body system is shown. At first the system collapses along the minor axis increasing its asphericity until, at maximum collapse it becomes sheet- or filament-like (see Figure 3(b)). Note that the collapse along the major axis is more chaotic. There is more time available for subclustering to develop and various subclumps are discernible just before maximum contraction along the major axis. At $t=60$ most of the system is 


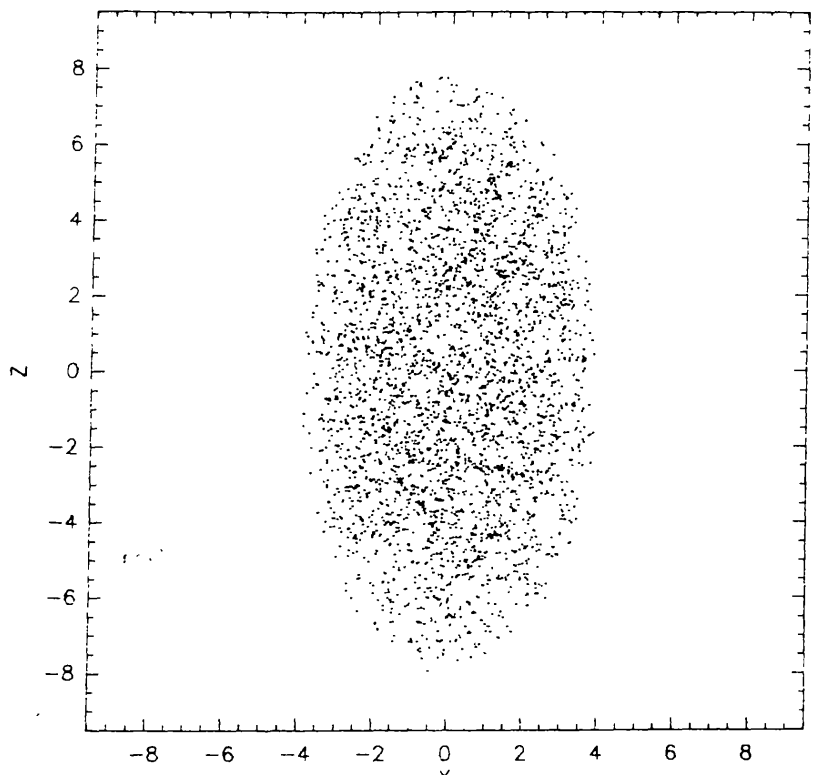

Fig. 4a.

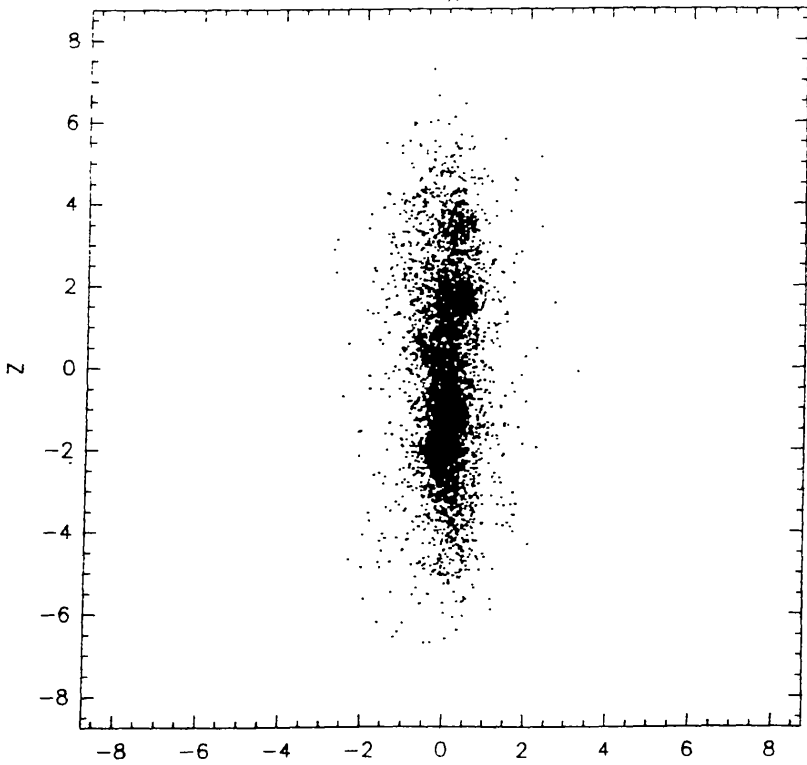

Fig. 4b.

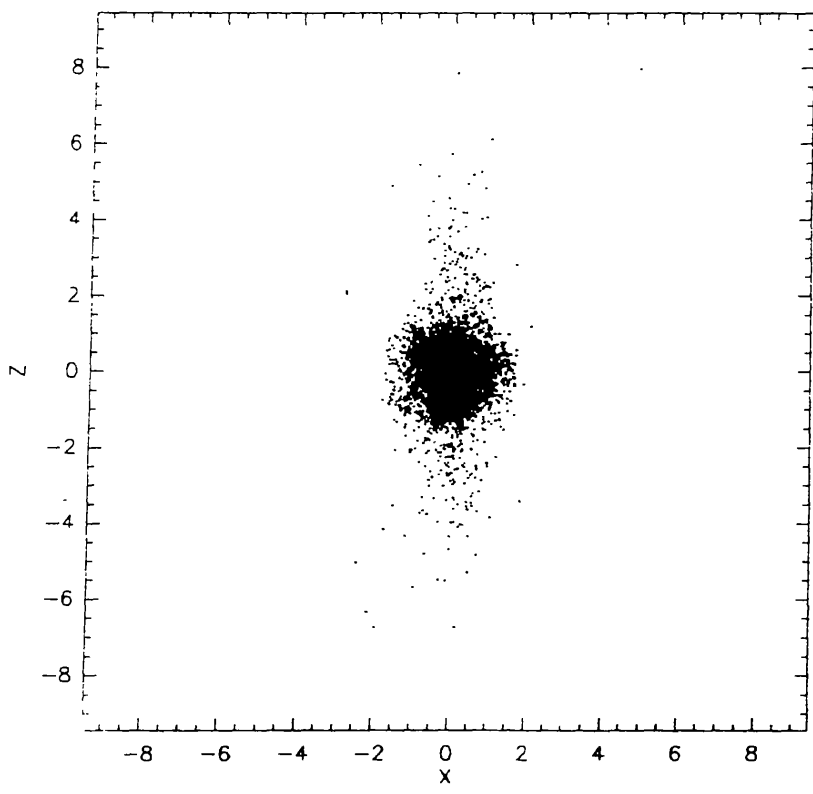

Fig. 4c. 


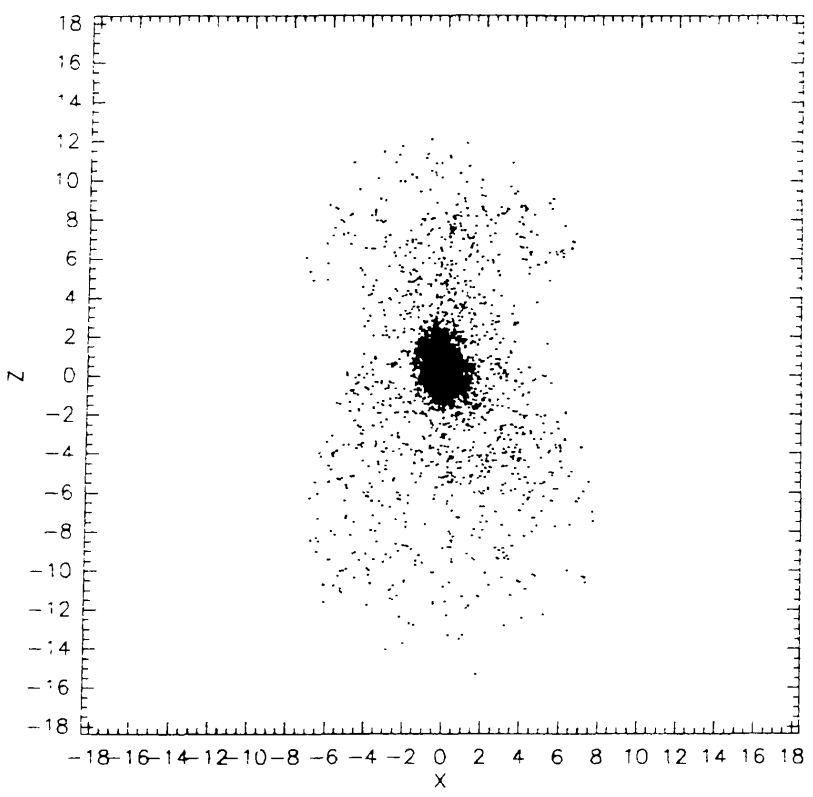

Fig. 4d.

Fig. 4. Particle distribution at (a) $t_{\text {out }}=0$, (b) $t_{\text {out }}=12$, (c) $t_{\text {out }}=16$, (d) $t_{\text {out }}=31$.

relaxed and virialized, but about one quarter of all particles is unbound or only slightly bound. For the latter particles phase mixing takes very long and, therefore, the outer envelope is not fully relaxed at the end of the simulation. In Figure 5 we give the projected number of particles in quarter shells along the major and minor axes. The shells are centred on the mean position of the most strongly bound 400 particles. The system is elongated over a factor 50 in radius, which is the maximum range we can cover in our simulations.

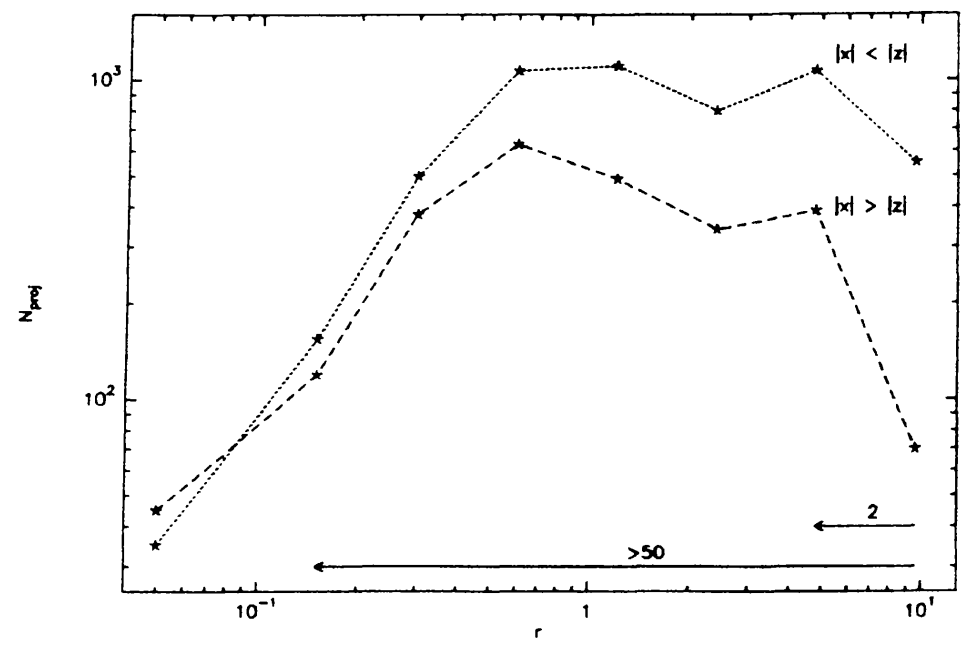

Fig. 5. Plot of the elongation of the system as function of radius as defined in the text. The system is elongated along the $x$-axis. We divide the plot into quadrants and radial bins. For each radial bin we sum the particles in opposing quadrants. The upper line corresponds to points with $|x|<|z|$ and the lower line to points with $|x|>|z|$. The fact that the number of particles with $|x|<|z|$ is consistently and significantly greater than the number of particles with $|x|>|z|$ over a large range in $r$ shows that the orientation is 'remembered' in the central part. 


\section{Discussion and Summary}

We have presented new evidence that first-ranked galaxies in rich clusters are aligned with their parent cluster and with the direction of the nearest neighbour cluster (scale $15 h^{-1} \mathrm{Mpc}$ ). The effect is stronger for $\mathrm{cD}$ and $\mathrm{gE}$ galaxies than for first-ranked galaxies of later Hubble type. This observation seems consistent with hierarchical clustering scenarios where most galaxies form before clusters. During the formation of clusters, substructure in the form of dissipationlessly formed galactic halos or subclusters merge while the smaller dissipationally formed luminous disk galaxies become detached or stripped from their halos as they acquire relative velocities with respect to the cluster centre of mass. Only those galaxies that partake in the merging process of the halos will remain attached to the common cluster halo. In small groups these galaxies may be identified with elliptical galaxies. It would be interesting to see if first ranked galaxies in small groups exhibit a similar orientation effect as expected in this model. During the formation of larger clusters most ellipticals will undergo the same fate as the disk galaxies and only the central giant elliptical or $\mathrm{CD}$ galaxy remembers the shape of the large-scale mass distribution. In this scenario cD galaxies are extreme members of the elliptical galaxy population (cf. Merritt, 1984), and, at the same time they can be regarded as the most successful cannibals (Hausman and Ostriker, 1978).

We are performing numerical simulations of the dissipationless collapse of systems having moderate asphericity. Our first results indicate that after virialization the central parts of such systems do indeed remember the initial large-scale anisotropy. For the moment we conclude that the orientation effect discussed above might be consistent with hierarchical clustering scenarios.

Finally we wish to point out that extrapolation of our result to galactic halos would imply that the central distribution of dark matter in halos has a similar shape to the distribution on larger scales. Dissipationally formed disk galaxies would then rotate in a triaxial potential. The disk's angular momentum vector, originating from the rotation of the outer halo, will generally not be perfectly aligned with that of the central part of the halo. This may have important implications for theories of spiral waves and for warps in isolated galaxies (Sparke, 1988).

\section{Acknowledgements}

We are grateful to $\mathbf{J}$. Barnes and P. Hut for making their code available to us and to P. Katgert for stimulating discussions and help with the figures. N. Roos wishes to thank the IAC for financial support of his participation in the Tenerife conference.

\section{References}

Aarseth, S. J. and Binney, J.: 1987, Monthly Notices Roy. Astron. Soc. 185, 227.

Argyres, P. C., Groth, E. J., Peebles, P. J. E., and Struble, M. F.: 1986, Astron. J. 91, 471.

Barnes, J.: 1985, in J. Goodman and P. Hut (eds.), 'Dynamics of Star Clusters', IAU Symp. 114, 297.

Beers, T. C. and Tonry, J. L.: 1986, Astrophys. J. 300, 557. 
Binggeli, B.: 1982, Astron. Astrophys. 107, 338.

Carlberg, R. G. and Couchman, H. N. P.: 1988, preprint.

Carter, D. and Metcalfe, N.: 1980, Monthly Notices Roy. Astron. Soc. 191, 325.

Cavaliere, A., Santangelo, P., Tarqini, G., and Vittorio, N.: 1986, Astrophys. J. 305, 651.

Djorgovski, S.: 1987, in S. Faber (ed.), Proceedings of the Santa Cruz Workshop 'Nearly Normal Galaxies', Springer-Verlag, Berlin.

Dressler, A.: 1980, Astrophys. J. 236, 351.

Flin, P.: 1987, Monthly Notices Roy. Astron. Soc. 228, 941.

Frenk, C. S., White, S. D. M., Davis, M., and Efstathiou, G.: 1988, Astrophys. J. 327, 507.

Hausman, M. and Ostriker, J.: Astrophys. J. 224, 320.

Icke, V.: 1972, 'Formation of Galaxies Inside Clusters', Ph.D. Thesis, Leiden University.

Icke, V.: 1984, Monthly Notices Roy. Astron. Soc. 206, 1P.

Lambas, D. G., Groth, E. J., and Peebles, P. J. E.: 1988, Astron. J. 95, 996.

Lynden-Bell, D.: 1964, Astrophys. J. 139, 1195.

McGlynn, T. A.: 1984, Astrophys. J. 281, 13.

Merritt, D.: 1984, Astrophys. J. 276, 26.

Merritt, D.: 1987, in T. de Zeeuw (ed.), 'Structure and Dynamics of Elliptical Galaxies', IAU Symp. 127, 315.

Ostriker, J. P. and Cowie, L. L.: 1981, Astrophys. J. 243, L127.

Rhee, G. F. R. N. and Katgert, P.: 1987, Astron. Astrophys. 183, 217.

Roos, N.: 1981, Astron. Astrophys. 95, 349.

Roos, N. and Aarseth, S. J.: 1982, Astron. Astrophys. 114, 41.

Sastry, G. N.: 1968, Publs. Astron. Soc. Pacific 80, 252.

Schombert, J. M.: 1988, Astrophys. J. 328, 475.

Sparke, L. S.: 1988, preprint.

Struble, M. F. and Peebles, P. J. E.: 1985, Astron. J. 90, 582.

Swaans, L.: 1981, Ph.D. Thesis, Leiden University.

Toomre, A. and Toomre, J.: 1972, Astrophys. J. 178, 623.

van Albada, T. S.: 1982, Monthly Notices Roy. Astron. Soc. 201, 939.

Villumsen, J. V.: 1984, Astrophys. J. 284, 75.

White, S. D. M. and Rees, M.: 1978, Monthly Notices Roy. Astron. Soc. 183, 341.

Wilkinson, A. and James, R. A.: 1982, Monthly Notices Roy. Astron. Soc. 199, 171. 